\title{
Role of Biofilm Production in Bacteria Isolated from Device Related and Non-Device Related Infection in a Tertiary Care Hospital
}

\author{
Gyaneshwar Tiwari $^{1 *}$, Bibhabati Mishra ${ }^{1}$, Vinita Dogra ${ }^{1}$ and D. R. Arora ${ }^{2}$ \\ ${ }^{1}$ Department of Microbiology, GIPMER, New Delhi 110002, India \\ ${ }^{2}$ SGT University, Gurugram, Haryana, India \\ *Corresponding author
}

\begin{tabular}{|l|}
\hline Ke y w o r d s \\
$\begin{array}{l}\text { Biofilm, Device } \\
\text { related, Non-device } \\
\text { related, Tissue } \\
\text { culture plate }\end{array}$ \\
\hline Article Info \\
\hline $\begin{array}{l}\text { Accepted: } \\
\text { 17 December } 2018 \\
\text { Available Online: } \\
\text { 10 January } 2019\end{array}$ \\
\hline
\end{tabular}

\begin{abstract}
A B S T R A C T
The use of indwelling devices both temporary and permanent, in medical and surgical practice has led to the emergence of implant associated infections in the patients admitted in a Tertiary care hospital leading to partial or complete therapeutic failure. The study was conducted to detect and compare biofilm production in bacteria isolated from device related (DR) and non-device related (NDR) infections by Tissue Culture Method (TCP). A total of 200 bacterial isolates from various DR and NDR clinical samples of patients suffering from hospital acquired infections were subjected to biofilm detection and drug susceptibility testing. Of the 200 strains in the present study 121 bacterial strains were isolated from device related and 79 from non-device related clinical samples. Of the DR isolates, $86(71.1 \%)$ were biofilm producers which included $10(11.6 \%)$ strong, 37 (43\%) moderate and $39(45.4 \%)$ weak producers whereas of the NDR isolates $66(83.5 \%)$ were biofilm producers including 18 (27.3\%) strong, 21 (31.8\%) moderate and, 27 (40.9\%) weak producers respectively. Device related biofilm producing strains of Klebsiella pneumoniae, Escherichia coli and Pseudomonas aeruginosa showed higher rate of drug resistance in comparison to their non-biofilm producing isolates. It is concluded that of bacteria isolates not only in device related infections but is also associated with multi drug resistance. Early detection of biofilm production will be of immense help in changing the modality of treatment with better patient outcome in device related infections.
\end{abstract}

\section{Introduction}

A Device related infection is defined as the host immune response to one or more microbial pathogens on an indwelling medical device. The use of indwelling devices both temporary and permanent, in medical and surgical practice has led to the emergence of implant associated infections. The association of biofilm and medical device related infections was first recognized in $1972 .{ }^{[1]}$ These infections include catheter- associated urinary tract infections (CAUTI), central-line-associated blood stream infections (CLABSI), and ventilatorassociated pneumonias (VAP). ${ }^{[2,3]}$

These infections are caused by bacterial colonization and biofilm formation on devices which help the microorganisms to acquire multiple antibiotic resistance and evade host immune response. Device related infections that unfortunately has received the least amount of attention, but which continues to 
contribute to major morbidity and mortality. ${ }^{[4]}$ Biofilm is an association of microorganisms in which microbial cells adhere to each other on a living or non-living surface within a self-produced matrix of extracellular polymeric substance. ${ }^{[5]}$

Biofilms on indwelling medical devices may be formed by gram-positive bacteria, gramnegative bacteria and yeasts. ${ }^{[6]}$ The predominant organisms responsible for device related infection are mostly Staphylococcus aureus (S aureus), Pseudomonas aeruginosa (Ps aeruginosa), and Enterobacteriaceae, but the etiologic agents differ widely according to the patient population in an intensive care unit, duration of hospital stay and prior antimicrobial therapy. ${ }^{[7-10]}$ These infections in turn lead to prolonged hospital stay along with increased burden of antibiotic usage, thereby leading to an overall increase in the health care cost. ${ }^{[11]}$

Very few studies have so far been reported in India as regards biofilm production by bacterial isolates in device related infections. In a study by Patel et al., vascular catheters and blood collected through catheter yielded the highest number of biofilm producing Gram negative bacilli. ${ }^{[12]}$ Most common pathogens isolated from device associated HAI patients were Klebsiella pneumoniae (K. pneumoniae) (24.6\%), Escherichia coli (E. coli) (21.9\%), and Ps aeruginosa (20.2\%). More than $80 \%$ of these strains were multidrug resistant. They were only susceptible to Colistin and Tigecycline. ${ }^{[13]}$

A prospective study has conducted over a period of one year (from April 2016 to March 2017). As this hospital is a tertiary care set up, indwelling medical devices are widely used in the various medical and surgery specialties. Commonly used medical devices are endotracheal tubes, tracheostomy tube, biliary stents, urinary catheters, CVC lines, peripheral venous catheters and drain tubes. No data is available in our institution regarding biofilm production by bacterial isolates in device related infections.

\section{Materials and Methods}

Various clinical samples from patients with device related hospital acquired infection, collected and processed as per Standard technique. Antimicrobial susceptibility testing has done by Kirby-Bauer disc diffusion technique as per CLSI guidelines 2016. ${ }^{\left[{ }^{[4]}\right.}$ The drug susceptibility was done for the following: Cephalosporins, Aminoglycosides, Fluoroquinolones, Penicillins, Macrolides, Glycopeptides, Oxazolidones, Cefoxitin and Carbapenams (Hi-media) were used for drug susceptibility testing. The control strains used were Esch coli ATCC 25922, Ps. aeruginosa ATCC 27853 and S. aureus ATCC 29213. Identification and drug susceptibility of the bacterial isolates was confirmed by the automated Vitek II Compact system. All these bacterial isolates were preserved for biofilm detection.

\section{Biofilm detection}

Biofilm detection was performed by the Tissue Culture Plate (TCP) method as described by Christensen et al., (1995), which is considered as the gold standard method. ${ }^{[15]}$ The bacteria were grown in polystyrene tissue culture plates for 24 hours. After washing fixed with sodium acetate $(2 \%)$ and stained with crystal violet $(0.1 \% \mathrm{w} / \mathrm{v})$. Biofilm formation was detected by measuring the optical density (OD) using ELISA reader. The experiment was performed in triplicate and repeated three times. The interpretation of biofilm production was done according to the criteria of Stepanovic et al., ${ }^{[16]}$

Data analysis was performed by Pearson ChiSquare test using SPSS software version 18. 


\section{Results and Discussion}

During the study period 219 bacterial strains were isolated from various clinical samples. Of these 200 isolates associated with hospital acquired infection (HAI) were included in the study. One hundred twenty one isolates $(60.5 \%)$ were device related (DR) and 79 $(39.5 \%)$ isolates were non-device related (NDR).

Details of device related and non-device related clinical sample is shown in table 1.

Maximum number of DR isolates were from respiratory samples (64) followed by drain fluid (42). While maximum NDR isolates were from pus (36) followed by blood samples (29).

Of the 121 bacterial strains were isolated from device related clinical samples maximum were $K$. pneumoniae 57(47.1\%) followed by Escherichia coli 28(23.1\%), Psaeruginosa 19(15.7\%), Acinetobacter baumannii (A baumannii) 11(9.1\%), $S$ aureus 3(2.5\%), Proteus mirabilis ( $P$ mirabilis) $2(1.7 \%)$ and Providencia species $1(0.8 \%)$. Of the 79 isolates from non-device related samples, highest were E. coli 22(27.9\%) followed by K.pneumoniae 21(26.6\%), S. aureus 12(15.2\%), A. baumannii 12(15.2\%), Ps. Aeruginosa 9(11.4\%), P. mirabilis 1(1.3\%), Burkholderia cepacia (B. cepacia) 1(1.3\%) and Enterobacter cloacae (E. cloacae) $1(1.3 \%)$.

Out of 200 MDROs, 152 isolates were biofilm producers and 48 were non-biofilm producers. Of 152 biofilm producing strains, 86wereDR while 66 were NDR isolates. Statistically significant difference was observed between DR and NDR biofilm production ( $p$ value is 0.04 ).

Of the 121 isolates from device related infections, $86(71.1 \%)$ strains were biofilm producers with maximum number being $A$. baumannii, $S$. aureus, $P$. mirabilis and Providencia spp. (100\%) followed by $K$. pneumoniae $(77.2 \%), \quad P$. saeruginosa (73.68\%) and E. coli $(39.28 \%)$.

$55.8 \%$ of the biofilm producers were associated with VAP followed by $32.6 \%$ Pyogenic infection, $8.1 \%$ CRI and $3.5 \%$ with CAUTI.

Of the 79 isolates from non-device related infections, $66(83.54 \%)$ strains were biofilm producers. Of these isolates all strains of A.baumannii, Ps. aeruginosa, P. mirabilis, $B$. cepacia and Enterobacter cloacae were biofilm producers $(100 \%)$ whereas $91.7 \%$ of $S$. aureus, $90.5 \%$ of $K$. pneumoniae and $54.5 \%$ of $E$. coli were found to be biofilm producers respectively. Among non-device related isolates $42.4 \%$ of biofilm producers were associated with pus followed by $39.4 \%$ blood stream infection (BSI), $12.1 \%$ respiratory and $6 \%$ body fluids.

Pus samples have the maximum number of biofilm producers 28(42.4\%) followed by Blood stream samples 26(39.4\%), respiratory samples $8(12.1 \%)$ and body fluids with the least number 4(6.1\%).

Biofilm production in (DR)/(NDR) isolates is shown in table 2 .

Among Gram negative isolates from both DR and NDR infections maximum of the biofilm producing strains isolated were $K$. pneumoniae (44 and 19 respectively).

Grading of biofilm producing isolates from device related /non- device related samples are shown in table 3.

Of the 86 biofilm producing DR isolates 10 were strong, 37 moderate and 39weak biofilm producers whereas among 66 biofilm 
producing NDR isolates 18, 21 and 27 were strong, moderate and weak biofilm producers respectively. Statistically significant difference was observed in grading of biofilm produced by DR and NDR isolates ( $p$ value is $0.04)$.

Drug resistance pattern (in percentage) of biofilm (BF) producers and non-biofilm (NBF) producers of DR isolates is depicted in table 4.

All strains of $A$. baumannii, $P$ mirabilis, Providencia spp. and S. aureus isolated from device related clinical samples were biofilm producers and were multi drug resistant
(MDR). Device related biofilm producing strains of K.pneumoniae showed higher rate of drug resistance in comparison to the nonbiofilm producing isolates against Piperacillin/Tazobactum (93\% and $77 \%)$, Ciprofloxacin (95\% and 77\%), Levofloxacin (79\% and 50\%), Imipenem (89\% and 69\%) Meropenem (91\% and 77\%) and Ertapenem (93\% and 58\%) respectively. Similar resistant pattern was observed in device related biofilm producing strains of $E$. coli and Ps.aeruginosa. NDR biofilm producing isolates showed almost similar resistance pattern as DR isolates. Biofilm producing (DR) $P s$ aeruginosa also showed 50\% resistance against Tigecycline.

Table.1 Clinical sample vs Device related (DR)/Non device related (NDR) isolates

\begin{tabular}{|c|c|c|}
\hline Clinical Samples & DR & NDR \\
\hline Respiratory sample & 64 & 9 \\
\hline Drain Fluid & 42 & 5 \\
\hline Tip & 10 & 0 \\
\hline Urine (Catheterized) & 5 & 0 \\
\hline Pus & 0 & 36 \\
\hline Blood & 0 & 29 \\
\hline Total & 121 & 79 \\
\hline
\end{tabular}

Table.2 Biofilm production in (DR)/(NDR) isolates

\begin{tabular}{|l|l|l|}
\hline BacterialIsolate (DR+NDR) & DR & NDR \\
\hline Klebsiellapneumoniae(57+21) & $44(77.2 \%)$ & $19(90.48 \%)$ \\
\hline Pseuodomonasaeruginosa(19+9) & $14(73.68 \%)$ & $09(100 \%)$ \\
\hline Escherichia coli(28+22) & $11(39.28 \%)$ & $12(54.55 \%)$ \\
\hline Acinetobacterbaumannii(11+12) & $11(100 \%)$ & $12(100)$ \\
\hline Staphylococcus aureus(3+12) & $3(100 \%)$ & $11(91.67 \%)$ \\
\hline Proteus mirabilis(2+1) & $2(100 \%)$ & $1(100 \%)$ \\
\hline Providenciaspp.(1+0) & $1(100 \%)$ & 0 \\
\hline Enterobacter Cloacae(0+1) & 0 & $1(100 \%)$ \\
\hline Burkholeriacepacia(0+1) & 0 & $1(100 \%)$ \\
\hline Total & $86(71.1 \%)$ & $66(83.54 \%)$ \\
\hline
\end{tabular}


Table.3 Grading of biofilm producing isolates from device related /non- device related samples

\begin{tabular}{|l|l|l|l|l|l|l|l|l|l|l|}
\hline \multirow{2}{*}{$\begin{array}{l}\text { Bacterial } \\
\text { Isolate name \& no. }\end{array}$} & \multicolumn{4}{|l|}{ Device related } & \multicolumn{4}{|l|}{ Non- device related } \\
\hline S & M & W & Total & N & S & M & W & Total & N \\
\hline Acinetobacterbaumannii(23) & 2 & 6 & 3 & 11 & 0 & 9 & 2 & 1 & 12 & 0 \\
\hline Pseuodomonas aeruginosa (28) & 1 & 7 & 6 & 14 & 5 & 2 & 1 & 6 & 9 & 0 \\
\hline Escherichia coli (50) & 1 & 2 & 8 & 11 & 17 & 2 & 4 & 6 & 12 & 10 \\
\hline Klebsiella pneumoniae(78) & 6 & 18 & 20 & 44 & 13 & 3 & 8 & 8 & 19 & 2 \\
\hline Staphylococcus aureus(15) & 0 & 3 & 0 & 3 & 0 & 1 & 5 & 5 & 11 & 1 \\
\hline Proteus mirabilis (03) & 0 & 1 & 1 & 2 & 0 & 0 & 0 & 1 & 1 & 0 \\
\hline Providencia spp. (01) & 0 & 0 & 1 & 1 & 0 & 0 & 0 & 0 & 0 & 0 \\
\hline Enterobacter cloacae (01) & 0 & 0 & 0 & 0 & 0 & 1 & 0 & 0 & 1 & 0 \\
\hline Burkholderia cepacia(01) & 0 & 0 & 0 & 0 & 0 & 0 & 1 & 0 & 1 & 0 \\
\hline Total & 10 & 37 & 39 & 86 & 35 & 18 & 21 & 27 & 66 & 13 \\
\hline
\end{tabular}

(*Abbreviations used for biofilm production: $\mathrm{S}=$ Strong, $\mathrm{M}=$ Moderate, $\mathrm{W}=$ Weak, $\mathrm{P}=$ Positive, $\mathrm{N}=$ Negative)

Table.4 Drug resistance pattern (in percentage) of biofilm (BF) producers and non-biofilm $(\mathrm{NBF})$ producers of $\mathrm{DR}$ isolates

\begin{tabular}{|c|c|c|c|c|c|c|c|c|c|}
\hline Antibiotics & $\begin{array}{l}\text { K.pnemoniae } \\
\text { BF } \\
(\mathrm{n}=44)\end{array}$ & $\begin{array}{l}\text { K.pnemoniae } \\
\text { NBF } \\
(\mathrm{n}=13)\end{array}$ & $\begin{array}{l}\text { E.coli } \\
\text { BF } \\
(n=11)\end{array}$ & $\begin{array}{l}\text { E.coli } \\
\text { NBF } \\
(n=17)\end{array}$ & $\begin{array}{l}\text { A.baumannii } \\
\text { BF } \\
(\mathrm{n}=11)\end{array}$ & $\begin{array}{l}\text { P.aeruginosa } \\
\text { BF } \\
(\mathrm{n}=14)\end{array}$ & $\begin{array}{l}\text { P.aeruginosa } \\
\text { NBF } \\
(\mathbf{n}=5)\end{array}$ & $\begin{array}{l}\text { P.mirabilis } \\
\text { BF } \\
(\mathrm{n}=2)\end{array}$ & $\begin{array}{l}\text { Providencia } \\
\text { Spp. BF } \\
(\mathrm{n}=1)\end{array}$ \\
\hline Ac & 98 & 92 & 94 & 72 & 100 & 100 & 86 & 100 & 100 \\
\hline Tz & 93 & 77 & 73 & 24 & 100 & 79 & 40 & 100 & 100 \\
\hline Co & 95 & 92 & 100 & 12 & 100 & 100 & 86 & 100 & 100 \\
\hline Cpm & 100 & 92 & 100 & 100 & 100 & 100 & 93 & 100 & 100 \\
\hline $\mathbf{C a}$ & 100 & 92 & 100 & 91 & 100 & 100 & 86 & 100 & 100 \\
\hline $\mathbf{C f}$ & 95 & 77 & 94 & 82 & 100 & 100 & 86 & 100 & 100 \\
\hline Of & 100 & 95 & 94 & 80 & 100 & 100 & 86 & 100 & 100 \\
\hline Le & 79 & 50 & 90 & 83 & 100 & 100 & 100 & 100 & 100 \\
\hline Akj & 100 & 75 & 41 & 25 & 91 & 75 & 71 & 100 & 100 \\
\hline $\mathbf{N t}$ & 100 & 83 & 71 & 63 & 100 & 100 & 86 & 100 & 100 \\
\hline Gm & 98 & 85 & 73 & 71 & 100 & 100 & 79 & 100 & 100 \\
\hline Tb & 98 & 92 & 82 & 71 & 100 & 83 & 80 & 100 & 100 \\
\hline Imp & 89 & 69 & 53 & 27 & 100 & 57 & 20 & - & 100 \\
\hline Mr & 91 & 77 & 59 & 55 & 100 & 80 & 77 & 0 & 100 \\
\hline Etp & 93 & 58 & 59 & 55 & 100 & 75 & 75 & 50 & 100 \\
\hline $\mathrm{Tg}$ & 25 & 23 & 6 & 0 & 18 & 50 & 30 & 0 & 0 \\
\hline CL & 30 & 23 & 14 & 12 & - & 23 & 0 & 0 & 100 \\
\hline
\end{tabular}

*Abbreviations:

$\mathrm{BF}=$ Biofilm producer, $\mathrm{NBF}=$ non-biofilm producers,

$\mathrm{n}=$ total no. of isolates,

$\mathrm{AC}=$ Amoxycilline + Clavulanic acid, $\mathrm{TZ}=$ Piperacillin+tazobactum, $\mathrm{CO}=$ Cotrimoxazole,

$\mathrm{CPM}=$ Cefpirome, $\mathrm{CA}=$ Ceftazidime, $\mathrm{CF}=$ Ciprofloxacin, $\mathrm{OF}=\mathrm{Ofloxacin}, \mathrm{Le}=$ Levofloxacin,

$\mathrm{AK}=$ Amikacin, $\mathrm{NT}=$ Netillmycin, $\mathrm{GM}=$ Gentamycin, $\mathrm{Tb}=$ Tobramycin, I=Imipenem,

$\mathrm{Mr}=$ Meropenem, Etp=Ertapenem, $\mathrm{Tg}=$ Tigecycline, $\mathrm{Cl}=$ Colistin.* 
Use of indwelling medical devices in patients admitted in the health care setup is colonized by biofilm producing organisms which are often multidrug resistant, thereby promoting drug resistant device-related infections. Medical device related infections not only pose huge financial burdens on the health care services but also prolong the course of the treatment thereby increasing morbidity and mortality.

The microorganisms are thus able to survive in the hospital environment despite unfavorable conditions such as desiccation, nutrient starvation and antimicrobial treatment. It is hypothesized that the microbes can persist in the environments and show high degree of virulence as a result of their capacity to colonize medical devices. ${ }^{[17]}$

Out of 200 strains in the present study 121 bacterial strains were isolated from device related and 79 from non-device related clinical samples. Of the device related isolates, $86(71.1 \%)$ were biofilm producers. Majority of biofilm producing bacteria included $P$. mirabilis, Providencia spp., A.baumannii and $S$ aureus, (100\%) followed by $K$. pneumoniae $(77.2 \%), P$. saeruginosa (73.68) and E. coli (39.28\%). In the study maximum number of biofilm producers were isolated from VAP (55.8\%) followed by Pyogenic infections (44.2\%), CRI (8.1\%) and CAUTI (3.5\%). Of the 86 biofilm producing device related bacterial isolates, $10(11.6 \%)$ were strong, 37(43\%) were moderate and 39 $(45.4 \%)$ were weak biofilm producers respectively.

Christensen et al., ${ }^{[15]}$ reported that $30 \%$ of biofilm forming bacteria were isolated from various indwelling medical devices, which is lower than the finding of the present study (71.1\%). The association of biofilm producing bacteria in urinary catheters was reported by Donalan (2001) in his study. ${ }^{[5]}$ According to a study by Hassan et al., ${ }^{[18]}$ the majority of the organisms associated with biofilm production were $S$. epidermidis $(37.1 \%)$ followed by $E$. coli $(27.1 \%), K$. pneumoniae $(15.7 \%), S$. aureus (11.4\%), E. faecalis (4.2\%) and $P$. aeruginosa (4.2\%). Of these biofilm producing bacteria $25.7 \%$ were isolated from urinary catheter tips followed by intravenous catheter tips (10\%). The findings of the present study is much higher than that of Hassan et al., but in both studies similar organisms were associated with device related infections. In a study conducted by Mulla et al., ${ }^{[19]}$ the overall biofilm production by bacterial isolates from patients with medical devices was $88 \%$ which is slightly higher in comparison to the results of the present study.

Pradeep Kumar et al., ${ }^{[20]}$ studied biofilm formation on 141 vascular catheters and 86 Foley catheters. They reported that $28 \%$ of the vascular catheters showed the presence of microbial biofilms and $80 \%$ of the Foley's catheter had microbial biofilms. Sayal et al., ${ }^{[21]}$ found that Escherichia coli was responsible for more than $80 \%$ of the UTIs. $71.23 \%$ of these isolates were found to be biofilm producers. Shyam et al., ${ }^{[22]}$ reported in a study that of the 67 clinical isolates from Indwelling Medical devices, $46.3 \%$ of the isolates were biofilm producers. According to a study of Patel et al., ${ }^{[12]}$ blood collected from Catheter showed the highest number of biofilm producers which includes Acinetobacter spp. (30\%), K.pneumoniae (22\%), Ps. aeruginosa (16\%), S. aureus (14\%) and E. coli (12\%). Most of the results are in agreement with the results of present study.

In a study by Singhai et al., ${ }^{[23]}$ the rates of biofilm-based catheter-related BSI, CAUTI, and VAP were $10.4 \%, 26.6 \%$, and $20 \%$ respectively. Majority of infections were due to K. pneumoniae followed by Staphylococcal biofilms. A high percentage of the biofilm 
producing bacterial isolates, were multidrug resistant and produced infections. Device related K.pneumoniae (73.1\%) were found to be the highest biofilm producers among device related isolates. ${ }^{[23]}$ Even in our study K.pneumoniae (77.2\%) was also one of the highest biofilm producers and drug resistant among DR isolates. Most of the studies mentioned above have findings similar to the present study.

Significant difference was observed in the production of biofilm and it's grading by bacterial isolates from device related and nondevice related clinical samples ( $p$-value $0.014)$

According to a study by Hassan et al., ${ }^{[18]}$ the majority of the organisms isolated from NDR clinical samples associated with biofilm production were S.epidermidis (37.1\%) followed by E. coli (27.1\%), K.pneumoniae (15.7\%), S. aureus (11.4\%), E. faecalis (4.2\%) and P. aeruginosa (4.2\%). Which is lower than the findings of the present study where Ps aeruginosa and A.baumannii showed maximum number of biofilm producers $(100 \%)$ followed by $S$ aureus $(91.67 \%)$ and K.pneumoniae (90.48\%). Maximum biofilm producing bacteria were isolated from urine (30\%) followed by, pus $(12.8 \%)$, sputum $(11.4 \%)$ and nasobronchial lavage specimens (10\%), whereas in the present study maximum of the isolates were from pus $(42.4 \%)$ followed by blood stream infection (39.4\%), respiratory samples (12.1\%) and body fluids $6.1 \%$ ).

In this study all strains of $A$. baumannii, $P$ mirabilis, Providencia spp. and $S$. aureus isolated from device related clinical samples were biofilm producers and multi drug resistant (MDR). Device related biofilm producing strains of $K$. pneumoniae showed higher rate of drug resistance in comparison to non-biofilm producing isolates. Similar resistant pattern was observed in device related biofilm producing strains of $E$. coli and $P$ s aeruginosa. Fifty percentages $(50 \%)$ of biofilm producing (DR) Ps. aeruginosa also showed resistance against Tigecycline. Biofilm producing isolates from non-device related infections showed almost similar drug resistance pattern as of DR isolates.

Singhai et al., ${ }^{[23]}$ reported in their study that a high percentage of biofilm producing bacterial isolates causing infection were multidrug resistant. Similar results were observed by Subramanian et al., ${ }^{[24]}$ in their study. Approximately $80 \%$ of the biofilm producing strains showed multidrug resistance. Shahidul et al., ${ }^{[25]}$ found that, $91.6 \%$ of the biofilm producing isolates were Multidrug resistant. All these studies are in agreement with present study.

The rate of drug resistance as seen in by biofilm producing isolates from device related samples is higher than that of the non-biofilm producing isolates in the present study.

In conclusion, the indwelling medical devices provide an ideal condition for the development of bacterial biofilms. These biofilms hinder the entry of antimicrobials and protecting the bacteria from their bactericidal effects thereby leading to increased morbidity and mortality. Thus these biofilm producers becomes MDR pathogens causing device related infections which often leads to partial or complete therapeutic failure.

Hence, biofilm production is an important virulence marker of bacteria isolates not only in device related infections but also associated with multi drug resistance. Biofilm associated bacteria from device related infection are more often MDR and this results in increased morbidity and mortality among the hospitalized patients. Early detection of 
biofilm production will be of immense help in changing the modality of treatment with better patient outcome in device related infections

\section{Acknowledgement}

I am very thankful to the Institutional research board for granting the permission for research and all faculty members of the department of microbiology for their help and support.

\section{References}

1. Johnson WG Jr., Pierce AK, Sanford JP, Thomas GD. Nosocomial respiratory infection with Gram negative bacilli; The significance of colonization of the respiratory tract. Ann Intern Med 1972; 77: 701-706.

2. Vincent JL, Bihari DJ, Suter PM, Bruining HA, White J, Nicolas-Chanoin $\mathrm{MH}$ et al., The prevalence of nosocomial infection in intensive care units in Europe. JAMA 1995; 274: 639-644.

3. American Thoracic Society, Infectious Diseases Society of America. Guidelines for the management of adults with hospital-acquired, ventilator-associated, and healthcare-associated pneumonia. Am J Respir Crit Care Med 2005; 171:388-416.

4. Darouiche RO. Device related infections. http//:www.infection controltoday.com posted on $30^{\text {th }}$ Nov. 2006.

5. Donlan RM. Biofilms and deviceassociated infections. Emerg Infect Dis 2001; 7(2): 277-81.

6. Stickler DJ. Bacterial biofilms and the encrustation of urethral catheters. Biofouling 1996; 94: 293-305

7. Pfaller MA, Diekema DJ. Epidemiology of invasive candidiasis: a persistent problem. Clin Microbiol Rev 2007; 20(1):133-163.

8. Pappas PG. Invasive candidiasis. Infect
Dis Clin North Am 2006; 20(3):485-506.

9. Sievert DM, Ricks P, Edwards JR,Schneider A, Patel J, Srinivasan A, et al. National Healthcare Safety Network (NHSN) Team and Participating NHSN Facilities, Antimicrobial-resistant pathogens associated with healthcareassociated infections: summary of data reported to the National Healthcare Safety Network at the Centres for Disease Control and Prevention 20092010. Infect Control Hosp. Epidemiol. 2013; 34(1):1-14.

10. Wisplinghoff $\mathrm{H}$, Bischoff $\mathrm{T}$, Tallent SM, Seifert H, Wenzel RP, Edmond MB. Nosocomial bloodstream infections in US hospitals: Analysis of 24,179 cases from a prospective nationwide surveillance study. Clin Infect Dis 2004; 39(3):309317.

11. Merle V, Germain JM, Bugel H, Nouvellon M, Lemeland JF, Czernichow $\mathrm{P}$, et al. Nosocomial urinary tract infections in urologic patients: assessment of a prospective surveillance program including 10,000 patients. EurUrol 2002; 41: 483-489.

12. Patel FM, Goswami PN, Khara R. Detection of biofilm formation in device associated clinical bacterial isolates in cancer patients. Sri Lankan Journal of Infectious Diseases. 2016 ;6(1): 43-50.

13. Khan ID, Basu A, Kiran S, Trivedi S, Pandit P, Chattoraj A. Device-Associated Healthcare-Associated Infections (DAHAI) and the caveat of multiresistance in a multidisciplinary intensive care unit. MJFI 2017; 73(3): 222-231.

14. Bauer AW, Kirby M M, Sherris JC, Jurek M. Antibiotic susceptibility testing by a standardized single method. Am J Clin Pathol 1966; 45:493-6.

15. Christensen GD, Simpson WA, Younger JJ, Baddour LM, Barrett FF, Melton DM, et al. Adherence of coagulase negative Staphylococci to plastic tissue cultures: a 
quantitative model for the adherence of Staphylococci to medical devices. J ClinMicrobiol 1995;22: 996-1006.

16. Stepanovic S, Vukovi D, Hola V, Di Bonaventura G, Djukić S, Cirković I,et al. Quantification of biofilm in microtiter plates: overview of testing conditions and practical recommendations for assessment of biofilm production by Staphylococci. APMIS 2007 115:891-9.

17. Gelosia A., Baldassarri L., Deighton M, Van Nguyen T. Phenotypic and genotypic markers of Staphylococcus epidermidis virulence. ClinMicrobiol Infect 2001;7:193-9.

18. Hassan A, Usman J, Kaleem F, Omair M, Khalid A, Iqbal M. Evaluation of different detection methods of biofilm formation in the clinical isolates. Braz $\mathbf{J}$ Infect Dis 2011; 15(4):305-311.

19. Mulla SA, Revdiwala S. Assessment of biofilm formation in device-associated clinical bacterial isolates in a tertiary level hospital. Indian J PatholMicrobiol 2011; 54: 561-4.

20. SSK Pradeep, HV Easwer, AM K N and. Multiple Drug resistant bacterial biofilms on implanted catheters- A reservoir of infection. J Assoc Physicians India Oct 2013; 61: 702-707.

21. Sayal P, Singh K, Devi P. Detection of bacterial biofilm in patients with indwelling urinary catheters. CIB Tech Journal of Microbiology 2014; 3(3):9-16.

22. Mishra SK, Basukala P, Basukala O, Parajuli K, Pokhrel BM, Rijal BP. Detection of Biofilm Production and Antibiotic Resistance Pattern in Clinical Isolates from Indwelling Medical Devices. CurrMicrobiol 2015;70(1):12834.

23. Singhai M, Malik A, Shahid M, Malik MA, Goyal R. A study on device-related infections with special reference to biofilm production and antibiotic resistance. J Glob Infect Dis 2012;4(4):193-8.

24. Subramanian P, Shanmugam N, Sivaraman UD, Kumar S, Selvaraj S. Antiobiotic resistance pattern of biofilmforming uropathogens isolated from catheterised patients in Pondicherry, India. Australas Med J. 2012; 5(7): 344348.

25. Shahidul KM, Asma A, Farahnaaz F, Sunjukta A. Determination of Antibiotic resistance pattern of Biofilm producing Pathogenic Bacteria associated with UTI. Int J Drug Dev Res 2013, 5 (4):312-319.

\section{How to cite this article:}

Gyaneshwar Tiwari, Bibhabati Mishra, Vinita Dogra and Arora, D. R. 2019. Role of Biofilm Production in Bacteria Isolated from Device Related and Non-Device Related Infection in a Tertiary Care Hospital. Int.J.Curr.Microbiol.App.Sci. 8(01): 2454-2464. doi: https://doi.org/10.20546/ijcmas.2019.801.259 